\title{
Aurora Kinase A (AURKA) Regulates The Progression and Imatinib Resistance of Advanced Gastrointestinal Stromal Tumors
}

\section{XIAOBIN CHENG ( $\sim 1512016 @ z j u . e d u . c n)$}

Zhejiang University School of Medicine https://orcid.org/0000-0002-2347-0318

\section{Jinhai Wang}

Zhejiang University School of Medicine

\section{Weina Fan}

Zhejiang University School of Medicine

Sen Lu

Zhejiang University School of Medicine

\section{Weilin Wang}

Zhejiang University School of Medicine

\section{Primary research}

Keywords: AURKA, Progression, Imatinib resistance, Gastrointestinal stromal tumors

Posted Date: April 12th, 2021

DOI: https://doi.org/10.21203/rs.3.rs-364465/v1

License: (c) (1) This work is licensed under a Creative Commons Attribution 4.0 International License. Read Full License

Version of Record: A version of this preprint was published at Cancer Cell International on July 31st, 2021. See the published version at https://doi.org/10.1186/s12935-021-02111-7. 


\section{Abstract}

Background: Gastrointestinal stromal tumor (GIST) is a common tumor that originates from mesenchyme in the alimentary system. Compared to the typical gastrointestinal carcinomas, GISTs exhibit unique malignant behaviors. Bioinformatic tools and subsequent experiments were applied to investigate novel targets involved in GIST progression and imatinib resistance.

Methods: Differences of gene expression profiles between advanced and non-advanced GISTs were comprehensively analyzed based on Gene Expression Omnibus (GEO) dataset GSE136755. A proteinprotein interaction (PPI) network was conducted to identify the potential target gene. Gene set enrichment analysis (GSEA) was used to elucidate relevant biological events of the target gene based on dataset GSE47911. Subsequently, immunohistochemistry and Kaplan-Meier analysis were performed to validate the prognostic value of the target gene in GISTs. Overexpression of the target gene was conducted to analyze its functions in proliferation, apoptosis, migration, and imatinib resistance of GIST/T1 cells.

Results: In current study, a total of 606 differentailly expressed genes (DEGs) were screened based on dataset GSE136755 and the upregulated DEGs in advanced GISTs were mainly involved in cell division through functional annotations. The intersected hub gene, Aurora Kinase A (AURKA), was identified by degree and bottleneck algorithms. GSEA revealed that AURKA was involved in cell cycle-related biological processes. Oncomine and GEPIA databases supported an elevated expression pattern of AURKA in most human malignances. Clinical assay demonstrated that AURKA could be an independent prognostic factor for GISTs. Additionally, overexpression of AURKA was experimentally demonstrated to promote cell proliferation and survival, and enhance imatinib resistance of GIST/T1 cells.

Conclusions: These findings indicated that overexpression of AURKA promoted GIST progression and enhanced imatinib resistance, implying the potential of AURKA as a therapeutic target for GISTs.

\section{Background}

Gastrointestinal stromal tumor (GIST) is the most common mesenchymal tumor of the alimentary system and originates from the interstitial cells of Cajal (ICC) $)^{[1]}$. About $71 \%$ of GISTs present with KIT $(71 \%)$ or PDGFRa $(14 \%)$ mutations, leaving approximately $10 \%-15 \%$ of GISTs without any KIT or PDGFRa mutations which are reported as KIT/ PDGFRa wild type ${ }^{[2,3]}$. The malignant potential of GISTs is stratified based on tumor size, mitotic index, and location according to the modified $\mathrm{NIH}$ criteria ${ }^{[4]}$. Based on these criteria, GISTs are classified as high-risk, intermediate-risk, low-risk, and very low-risk. The 5-year survival rate of advanced GIST patients is between $35 \%$ and $65 \%{ }^{[5]}$. The main therapeutic option for primary localized GISTs is surgical resection. However, the recurrence rate for GISTs, even after complete surgical resection, is $40 \%-80 \%{ }^{[5]}$. The median time to recurrence for most patients is approximately 12 -16 months $^{[6]}$. Treatment with imatinib, a tyrosine kinase inhibitor (TKI) that targets KIT and PDGFRa, has improved the prognosis of GIST patients. However, when used to eliminate mature GIST cells, imatinib has a limited efficacy, and studies have suggested GIST persistence in prolonged TKI therapy ${ }^{[1]}$. Due to 
acquired resistance to imatinib, about $85 \%-90 \%$ of GIST patients usually experience disease progression within $20-24$ months ${ }^{[2,3,7]}$. Further researches are urgently needed to reveal the mechanism of GIST progression and explore novel therapeutic targets for imatinib resistance.

Currently, bioinformatic tools are being used to evaluate molecular signatures associated with progression and clinical outcomes in several types of malignancies ${ }^{[8-10]}$. In this study, the datasets GSE136755 and GSE47911 downloaded from the Gene Expression Omnibus (GEO) were used to evaluate the potential target genes involved in GIST progression. Among several hub genes in the dataset GSE136755, AURKA was considered as a key hub gene in GIST progression. Gene set enrichment analysis (GSEA) based on dataset GSE47911 recommended that AURKA appeared to promote GIST progression by regulating cell cycle processes. Subsequent clinical data analyses demonstrated the value of AURKA as a prognostic factor for GISTs. Furthermore, overexpression of AURKA was experimentally demonstrated to significantly promote cell proliferation and survival, and enhance imatinib resistance of GIST/T1 cells.

\section{Materials And Methods}

\section{Data acquisition and differentially expressed genes (DEGs) identification}

The dataset GSE136755 was downloaded from the Gene Expression Omnibus (GEO) (https://www.ncbi.nlm.nih.gov/geo/) ${ }^{[11]}$. The GSE136755 is based on the GPL17077 platform (Agilent039494 SurePrint G3 Human GE v2 8x60K Microarray 039381), and includes clinicopathological information for 65 human GIST tumor samples without preoperative imatinib treatment. Primary GISTs with KIT mutations were selected to screen the differentially expressed genes (DEGs). High-risk GISTs were defined as advanced samples (16 samples), while low-risk and very low-risk GISTs were considered as non-advanced samples (31 samples). Intermediate-risk GISTs were not included because they were not obviously special in terms of biological behaviors compared to the high-risk and low-risk GISTs. GEO2R, an R-associated web tool from the National Center for Biotechnology Information, was used to screen the DEGs between advanced and non-advanced GISTs ${ }^{[12]}$. The DEGs were identified using the cutoff values of: |log2FoldChangel $>1$ and adju. $p<0.05$. For hierarchical clustering analysis in Morpheus (https://software.broadinstitute.org/morpheus), the DEGs were reserved in text format.

\section{Functional enrichment analysis of DEGs}

The Database for Annotation, Visualization, and Integrated Discovery (DAVID) (http://david.ncifcrf.gov, version 6.8) is a web based bioinformatics resource that is used to extract functional annotation information of genes ${ }^{[13]}$. Gene ontology $(\mathrm{GO})$ is a major bioinformatics tool for gene annotation and analysis ${ }^{[14,15]}$. The Kyoto Encyclopedia of Gene and Genome (KEGG) is a popular database for the analysis of advanced gene functions and potential signaling pathways in large-scale molecular data ${ }^{[16,}$ 17]. DAVID was used to perform GO and KEGG enrichment analyses of DEGs. The cut-off criterion was a false discovery rate $($ FDR $)<0.05$. 


\section{Protein-protein interaction (PPI) network and module analysis}

The Search Tool for the Retrieval Interacting Genes (STRING) (http://string-db.org) is an online database that is used to identify interactions among DEGs ${ }^{[18,19]}$. A confidence score $\geq 0.7$ was set for conducting the PPI network. Cytoscape (version3.7.1), is an open-source bioinformatics software platform that is used for visualizing the PPI network and for further analyses ${ }^{[20,21]}$. The plugin-in Molecular Complex Detection (MCODE) of Cytoscape was performed to identify significant modules based on the PPI network topology. The criteria were: degree cut-off $=2$, node score cut-off $=0.2, \mathrm{~K}$-core $=2$, and Max. depth $=100$. Plug-in apps, ClueGO, were used to analyze and visualize the biological processes and pathways in significant modules.

\section{Hub gene identification and analysis}

Plugin-in cytoHubba of Cytoscape was used to identify hub genes based on the degree and bottleneck algorithms. The Oncomine database is an online platform that computes gene expression signatures, clusters, and gene-set modules ${ }^{[22]}$. Gene Expression Profiling Interactive Analysis (GEPIA) database is a newly developed web server for cancer, normal gene expression profiling and interactive analyses ${ }^{[23]}$. The cytoHubba was used to identify the hub genes, whose expression patterns were evaluated in common human malignancies using the Oncomine and GEPIA databases.

Clinicopathological features and KIT/PDGFRa mutation types were extracted from GSE136755 and from the raw data provided by Lagarde et al. ${ }^{[24]}$ Correlations between key gene expression patterns and clinicpathological features as well as KIT/PDGFRa mutation types were statistically determined, with a $\mathrm{P}<$ 0.05 set as the threshold for statistical significance.

\section{Gene set enrichment analysis (GSEA)}

GSEA is a computational method that determines whether a priori defined gene set shows statistically significant and concordant differences between two biological states ${ }^{[25,26]}$. The GSEA computes biological information from different perspectives and further elucidates on relevant biological events. The dataset GSE47911, based on the GPL6480 platform (Agilent-014850 Whole Human Genome Microarray 4x44K G4112F), was also downloaded for the GEO database. This dataset has 15 gastric GIST samples ${ }^{[27]}$. Based on the key gene expression level, GIST samples were divided into two groups, after which GSEA was subsequently performed. Annotated gene sets (c2. cp.kegg.v7.2.symbols.gmt [Curated], c2.cp.ractome.v7.2.symbols.gmt [Curated], and c5.bp.v7.2.symbols.gmt. [Gene oncology]) were chosen as the reference gene sets. Gene size $>20, F D R<0.05$ and normalized enrichment score (NES) > 2.00 were set as the cut-off criteria.

\section{Immunohistochemistry and survival analysis}

Between 2001 and 2015, a total of 49 patients admitted to the First Affiliated Hospital of Zhejiang University (Zhejiang Province, China), who were diagnosed with GISTs, were enrolled in this study. None 
of the patients had incomplete resection, neoadjuvant and adjuvant imatinib treatment, and a family history of GISTs. Clinical stratification of GISTs was based on the modified NIH criteria ${ }^{[4]}$. Paraffinembedded GIST samples were obtained from the study participants and used to perform immunohistochemical (IHC) staining as well as survival analysis. All GIST tissue samples were provided by the Department of Pathology, the First Affiliated Hospital of Zhejiang University. The use of human tumor samples and clinical data in this study was approved by the Ethical Committee, the First Affiliated Hospital of Zhejiang University. Study participants were required to sign a written informed consent before enrollment. IHC staining was performed as previously described ${ }^{[28]}$. Briefly, tissue sections were incubated at $4{ }^{\circ} \mathrm{C}$ overnight with anti-human AURKA rabbit polyclonal antibody diluted 1:500 (NOVUS Biologicals, USA). A total of five adjacent fields using $\times 400$ magnifications in areas with the highest density of positive staining were scored according to the summation of the percentage of staining intensity. The immunostaining percentage was defined as $0(<5 \%), 1(<20 \%), 2(20-50 \%)$, and $3(>50 \%)$. Staining intensity was defined from 0 (no staining) to 3 (strongest staining). The maximum score of $\mathrm{IHC}$ staining was 6 , corresponding to more than $50 \%$ of the cells with the strongest staining intensity. The staining scores of less than the mean value were considered low expression, while scores more than the mean value were considered high expression. The follow-up time for all the patients was calculated from the surgical date to disease recurrence or last visit dates.

\section{Cell culture}

The GIST cell line (GIST/T1) donated by Prof. Wenbin Chen (Zhejiang University, Hangzhou, China), was obtained from the Cell Bank of the Type Culture Collection of the Chinese Academy of Sciences (Shanghai, China). Cells were maintained in RPMI-1640 medium (GIBCO) supplemented with $10 \%$ heatinactivated fetal bovine serum (GIBCO) in a humidified atmosphere with $5 \% \mathrm{CO}_{2}$ at $37^{\circ} \mathrm{C}$.

\section{Construction and transfection of lentiviral vectors for AURKA}

The DNA fragment encoding the AURKA sequence was synthesized and inserted into the lentivirus expression vector, pLVX-IRES-tdtomato (TaKaRa, China). The resulting vector was identified as pLVXAURKA-IRES-tdtomato. Lentiviral plasmids were transfected into HEK 293T cells with psPAX2 and pMD2.G at a ratio of 4:3:1 using Lipofectamine 2000 (Invitrogen, USA). Then, 48 hours after transfection, the virus was isolated. GIST/T1 cells were infected with lentivirus for $48 \mathrm{~h}$, and the transfection efficiency was assessed by PCR and western blotting.

\section{RNA extraction and real-time quantitative PCR (RT-qPCR)}

Total RNA was extracted using the TRIzol reagent (Generay Biotech, China) according to the manufacturer's instructions. The extracted RNA was then treated with RQ1 RNase-Free DNase (Promega, USA). Then, reverse transcription was performed using PrimeScriptTM RT Master Mix (Takara, China) according to the manufacturer's instructions. RT-qPCR analysis was performed to confirm the expression levels of AURKA using CFX Connect Real-Time System (BIO-RAD, USA) with the SuperReal PreMix Color 
SYBR Green kit (Tiangen, China). The primer sets used for RT-qPCR are shown in Table 1. Gene expression levels were normalized against the internal control using the $2^{-\triangle \Delta C T}$ method.

\section{Western Blotting}

Protein lysates from each sample were separated by $10 \%$ sodium dodecyl sulfate-polyacrylamide gel electrophoresis (SDS-PAGE), and transferred to a polyvinylidene difluoride (PVDF) membrane (Merck Millipore, USA). The membrane was blocked with $5 \%$ non-fat milk or $5 \%$ BSA in TBST (tris-buffered saline with $0.1 \%$ Tween 20 ) for $1 \mathrm{~h}$ at room temperature. Then, the membrane was incubated with a primary antibody AURKA (1:1000 dilution, NOVUS Biologicals, USA) overnight at $4{ }^{\circ} \mathrm{C}$, washed thrice using $0.1 \%$ TBST buffer for $30 \mathrm{~min}$, probed with Goat anti-Rabbit IgG-HRP secondary antibody (1:2000 dilution; Santa Cruz, USA), and washed thrice using $0.1 \%$ TBST buffer for $30 \mathrm{~min}$. The HRP conjugated secondary antibody was detected and visualized using the enhanced chemiluminescence detection system (GE Healthcare, USA). Band intensity was quantified by densitometry using the ImageJ software (version 1.49; National Institutes of Health, USA) ${ }^{[29]}$.

\section{Proliferation assay}

Cell proliferation was assessed by the CCK8 assay (Tongren Chemical Society, Japan). Briefly, $1 \times 10^{5}$ cells per well were seeded in 96-well plates and incubated in a $5 \% \mathrm{CO}_{2}$ atmosphere at $37^{\circ} \mathrm{C}$ for $24 \mathrm{~h}$. Imatinib $(3 \mu \mathrm{M})$ was then added to the culture medium for $48 \mathrm{~h}$ to evaluate the effect of AURKA on imatinib resistance. The medium was discarded, replaced with serum-free medium as well as CCK8 (10 $\mu \mathrm{l})$ and incubated for $2 \mathrm{~h}$. The Biokinetics Reader (MD corporate, USA) was used to detect absorbance at $450 \mathrm{~nm}$.

\section{Apoptosis assay}

Cell apoptosis was assessed using the Annexin V-APC/7-AAD apoptosis kit (MultiSciences, China), according to the manufacturer's instructions. Briefly, $3 \times 10^{5}$ cells per well were seeded in 6-well plates and incubated in a $5 \% \mathrm{CO}_{2}$ atmosphere at $37^{\circ} \mathrm{C}$ for $24 \mathrm{~h}$. Imatinib $(3 \mu \mathrm{M})$ or solvent, were added to the culture medium for $48 \mathrm{~h}$, after which the cells were collected. After washing twice in PBS at $4^{\circ} \mathrm{C}$, cells were re-suspended in a binding buffer $(500 \mu \mathrm{l})$. Annexin V-APC $(5 \mu \mathrm{l})$ and 7-AAD $(10 \mu \mathrm{l})$ were added into the suspension and incubated for $5 \mathrm{~min}$ at $4^{\circ} \mathrm{C}$. The apoptosis index was examined by flow cytometry (ACCURI C6; BD, USA).

\section{Migration assay}

Cell migration was assessed in 24-well trans-well chambers with upper and bottom culture compartments separated by a polycarbonate membrane with $8 \mu \mathrm{m}$ pore size. $7.5 \times 10^{4}$ cells at logarithmic stage were seeded in the upper chamber. The culture medium with/without imatinib $(3 \mu \mathrm{M})$, was added to the bottom chamber. Cells were incubated for $72 \mathrm{~h}$ and later fixed in $4 \%$ paraformaldehyde. Non-migrated cells were washed and the inserts air-dried. Then, cells were incubated in $200 \mu \mathrm{l}$ of $1 \%$ crystal violet for 10 min at 
room temperature. The number of cells that invaded the gel were counted in 3 fields $(\times 100)$ using an inverted microscope (IX73; OLYMPUS, Japan).

\section{Statistical analysis}

All statistical analyses were performed using the SPSS 25.0 software (SPSS Inc., USA). Descriptive data were expressed as mean \pm SD. Comparison of more than two mean values was performed by one-way analysis of variance (ANOVA) while the student's $t$-test was used to compare two mean values. KaplanMeier analysis was performed to establish disease-free survival (DFS) curves, while the log-rank test was used for survival curve comparison. The COX proportional hazards model was then used to perform multivariate analysis, while the Forward Likelihood Ratio method was used to identify independent variables. $P<0.05$ was set as the threshold for statistical significance.

\section{Results}

\section{Identification of differentially expressed genes (DEGs)}

A total of 65 human GIST samples without preoperative imatinib treatment were included in dataset GSE13675. A cohort of 47 samples with KIT mutations, consisting of 16 advanced (high-risk) GIST patients and 31 non-advanced (low-risk and very low-risk) GIST patients, was used to screen the DEGs using GEO2R. A total of 606 genes (244 upregulated and 362 downregulated) were identified using the cut-off criteria of adj. $p<0.05$ and Log2FoldChange $>1$. The top 50 up-and down-regulated genes were presented in a heatmap (Figure 1A, supplementary file 1).

\section{Functional annotation of DEGs}

To evaluate the biological clustering of DEGs, GO and KEGG analyses for the up- and downregulated DEGs were performed using DAVID. Based on GO analysis, upregulated DEGs were found to be significantly enriched in cell division, sister chromatid cohesion, mitotic nuclear division, microtubulebased movement, and mitotic metaphase plate congression. Cellular component (CC) of the upregulated DEGs was significantly enriched in midbody, kinesin complex, spindle, spindle microtubule, and condensed chromosome kinetochore. Molecular function (MF) of the upregulated DEGs was significantly enriched in microtubule motor activity and microtubule-binding. KEGG analysis showed that the upregulated DEGs were mainly involved in cell cycle and oocyte meiosis. GO analysis revealed that the down-regulated DEGs were significantly enriched in the interferon-gamma-mediated signaling pathway, type I interferon signaling pathway, and antigen processing and presentation of antigens. The CC of the down-regulated DEGs was significantly enriched in the integral component of the lumenal side of the endoplasmic reticulum membrane and ER to Golgi transport vesicle membrane while the MF of the downregulated DEGs was significantly enriched in peptide antigen binding and MHC class II receptor activity. KEGG analysis further revealed that the down-regulated DEGs were mainly involved in Graft-versus-host disease, Type I diabetes mellitus, Allograft rejection, Antigen processing and presentation, and Viral myocarditis. These results are presented in Figure 1B (supplementary file 2). 


\section{Module analysis through the PPI network of DEGs}

To clarify the DEGs functionally, STRING was used to conduct a PPI network, which was composed of 603 nodes and 1768 edges. The PPI enrichment $p$-value was $<1.0 \mathrm{E}-16$. The PPI network was visualized by Cytoscape and further analyzed by plug-in MCODE. Two most significant modules (module 1 and module 2) were identified and analyzed using GO, KEGG and REACTOME annotations to infer their biological functions. The module 1 (MCODE score $=36.667$ ) was mainly involved in in cell cycle-related biological processes and signaling pathways, while the module 2 (MCODE score $=18.759)$ was mainly involved in immunological processes and signaling pathways. These results are presented in Figure $2 \mathrm{~A}$ (supplementary file 2).

\section{Identification of hub genes}

To reveal the crucial genes underlying the regulation of GIST progression, we filtered hub genes among DEGs using the plugin-in cytoHubba of Cytoscape. Two algorithms, degree and bottleneck, were applied to weight the DEGs. The degree algorithm calculates the relevance and abundance of genes, while the bottleneck algorithm evaluates key genes positions in an entire regulatory network. According to the degree algorithm, the top 15 hub genes were CDK1, KIF11, KIF2C, CENPE, KIF20A, BUB1, CCNA2, CCNB1, AURKA, MAD2L1, CDCA8, KIF4A, CENPF, NDC80, and KIF23, with their scores ranging from 58 to 48. According to the bottleneck algorithm, the top 15 hub genes were AURKA, FN1, CD44, VEGFA, IL6, HLADQB1, HLA-DPA1, CXCL8, NT5E, ANK2, FOXM1, CHEK1, STAT1, CDC25A, and IFIH1, with their scores ranging from 64 to 8 . A Venn diagram was used to identify the key hub genes by taking intersection of the two hub gene cohorts. The result showed that AURKA was the only overlapping hub gene (Figure 2B).

\section{Gene Set Enrichment analysis (GSEA)}

A total of 15 human gastric GIST samples in GSE47911 includes 6 high-risk cases, 1 intermediate-risk case, 3 low-risk cases, and 5 very low-risk cases. To further verify significant biological processes associated with AURKA expression, GSE47911 gene profiles were divided into two groups after which GSEA was performed based on AURKA expression level. Samples with the highest ( $25 \%, 4$ samples) and lowest (25\%, 4 samples) expression levels were selected for further analysis using GSEA. Cell cyclerelated gene sets were associated with elevated AURKA expression (Figure 2C, supplementary file 3 ).

\section{Correlation between AURKA expression and clinicopathological features in GISTs}

To evaluate the clinical significance of AURKA expression in GISTs, AURKA expression levels in 49 GIST tissues were assessed by IHC staining (Figure 3A). The correlations between AURKA expression and clinicopathological features (age, gender, location and risk stratification) were determined (Table 2, supplementary file 4). AURKA expression was closely associated with tumor risk stratification (Figure 3B; $P<0.001)$. The clinical significance of AURKA expression in GISTs was also evaluated using the data from GSE136755 and the raw data provided by Lagarde et al. ${ }^{[24]}$ (Table 3, Table 4, and Figure 4, supplementary file 5). Findings from the GSE136755 revealed significant associations between AURKA 
expression and tumor risk stratification $(P<0.001)$ as well as tumor stage $(P<0.001)$, while analyses of the raw data from Lagarde et al. ${ }^{[24]}$ also showed a significant association between AURKA expression and tumor risk stratification $(P<0.001)$ as well as tumor recurrence $(P<0.001)$ and metastasis $(P<$ 0.001). However, apart from GSE136755 which revealed a significant association between AURKA expression and tumor location $(P=0.018)$, the data provided by Lagarde et al. did not establish a significant association between AURKA expression and tumor location $(P=0.156)$.

To determine the prognostic value of AURKA expression in GISTs, Kaplan-Meier survival analysis was performed. The range of observation time was 9 - 79 months. As shown in Figure 3C, GIST patients with elevated AURKA expression exhibited poorer DFS than those with low AURKA expression level (43.25 \pm 6.94 months vs $98.48 \pm 3.44$ months $₫ P<0.001)$. COX proportional hazard model showed that AURKA could be used as an independent prognostic marker for GISTs $(P=0.002)$.

Gene mutation types can predict the responsiveness of GISTs to imatinib. GISTs with KIT exon 11, PDGFRa exon 12 and PDGFRa exon 14 mutations were considered sensitive to imatinib. GISTs with other mutations, such as KIT exon 9, KIT exon 13, KIT exon 14, KIT exon 17, KIT exon 18, PDGFRa exon 18 D842V and KIT/PDGFRa wild type GISTs, were insensitive/resistant to imatinib ${ }^{[1,3,30]}$. In the dataset GSE136755, containing 56 imatinib-sensitive and 7 imatinib-resistant GISTs, there was a weak association between AURKA expression and imatinib-resistant gene mutations (Figure 4A, $P=0.074$ ). Through analysis of the raw data provided by Lagarde et al. ${ }^{[24]}$, which contained 45 imatinib-sensitive and 15 imatinib-resistant samples, it was shown that there was a strong association between AURKA expression and imatinib-resistant gene mutations (Figure 4B, $\mathrm{P}=0.018$ ).

\section{AURKA expression patterns in common human malignancies}

To determine whether elevated AURKA expression is common in human digestive malignancies, mRNA expression levels of AURKA in stomach carcinoma, liver hepatocellular carcinoma, and colorectal carcinoma were evaluated using data from the GEPIA database. AURKA expression was found to be significantly up-regulated in all the above malignancies by comparison to the normal tissues. Findings from the Oncomine database also supported the upregulated expression of AURKA in most human malignancies (Figure 3D).

\section{AURKA overexpression enhances GIST/T1 cell proliferation, survival, and migration}

To assess the biological effects of AURKA expression in GISTs, AURKA was overexpressed in GIST/T1 cells transfected with the AURKA-expressing virus, which was defined as the AURKA overexpression group (AURKA group). Normal GIST/T1 cells (Blank group) and GIST/T1 cells transfected with vacant plasmids (Vectorgroup) were considered as the control groups. The transfection efficiency was determined by the red fluorescence from tdtomato, and quantified by RT-qPCR and western blotting. Figure 5 showed that compared to the Blank and Vectorgroups, AURKA was overexpressed in GIST/T1 cells transfected with AURKA-expressing virus (the AURKA group). (supplementary file 6) 
The CCK8 assay was performed to assess the effect of AURKA overexpression on cell proliferation. Compared to the Blank and Vectorgroups, the overexpression of AURKA significantly enhanced GIST/T1 cell proliferation $(P=0.018$ ) (Figure $6 A)$. Imatinib treatment significantly inhibited cell proliferation in all the three groups. However, compared to the control groups, AURKA overexpressed cells still showed a relatively higher proliferation rate in the presence of imatinib $(P<0.001$, Figure $6 A)$. (supplementary file 6$)$

We also established that AURKA overexpression markedly suppressed the apoptotic process in GIST/T1 cells $(P<0.001$, Figure $6 B)$. Similar result was observed in the circumstance of imatinib administration. Compared to the Blank and Vectorgroups, AURKA overexpression obviously inhibited cell apoptosis after imatinib administration $(P<0.001$, Figure 6B). The results suggested that the AURKA overexpression enhanced resistance of GIST cells to imatinib. (supplementary file 6)

Apart from cell proliferation and apoptosis, the effect of AURKA overexpression on cell migration was also evaluated. The number of AURKA overexpressed cells that passed through the membrane was significantly higher than that of the other two control groups (for $A U R K A$ group, rate $=47.00 \pm 5.06 \%$; for Blank group, rate $=26.17 \pm 3.66 \% \bigotimes$ for Vectorgroup, rate $=30.00 \pm 4.15 \%)$ in the absence of imatinib $(P<$ 0.001 , Figure $6 C)$. However, imatinib significantly suppressed cell migration in all three groups $(P<0.001$, Figure $6 \mathrm{C})$ and effectively eliminated the contribution of AURKA overexpression to cell migration $(\mathrm{P}=$ 0.169 , Figure 6C). (supplementary file 6)

\section{Discussion}

GIST is a common mesenchymal malignancy of the human alimentary system. Compared to gastrointestinal carcinomas, GIST is known to possess special biological features. For example, lymph node metastasis is not common in GIST and occurs preferably in young patients ${ }^{[31,32]}{ }^{\text {. A } 1 \text { - or } 2-c m}$ macroscopic margin may be sufficient to achieve microscopically negative margins ${ }^{[33]}$. GIST responds poorly to conventional chemotherapy and radiotherapy ${ }^{[34]}$. As such, to better understand GIST biological behavior and inform the development of therapeutic strategies, it is conductive to establish the crucial genes that regulate the malignant behavior of GIST. Bioinformatics advances have been useful in exploring molecular targets for the progression and prognosis of $\operatorname{GIST}^{[11,35,36]}$.

In this study, gene profiles of 47 GIST samples from the dataset GSE136755 were selected for further analyses. Comparison of the gene profiles between advanced and non-advanced GISTs generated 244 upregulated DEGs and 362 downregulated DEGs. Functional annotation based on GO and KEGG analyses showed that upregulated DEGs were mainly enriched in cell cycle-related biological processes and signaling pathways, while the downregulated DEGs were mainly enriched in immune-related biological processes and signaling pathways. STRING database and Cytoscape software were used for further exploration of DEGs. Two important modules were extracted and visualized. Module 1 was consisted of upregulated DEGs and mainly involved in cell cycle-related biological processes and signaling pathways, while module 2 was consisted of downregulated DEGs and mainly involved in 
immunological processes and signaling pathways. This indicates that the difference in gene profiles between advanced and non-advanced GISTs is mainly reflected in cell cycle and tumor immunity.

Based on the degree and bottleneck algorithms, plug-in cytoHubba in Cytoscape software was used to screen for novel key genes associated with GIST progression. The degree algorithm calculates the relevance and abundance of genes, while the bottleneck algorithm evaluates key gene positions in an entire regulatory network. In this study, a significant key gene, AURKA, was identified using a Venn diagram. AURKA is a protein-coding gene that encodes a cell cycle-regulated kinase involved in microtubule formation and/or stabilization at the spindle pole during chromosomal segregation. It has been documented that AURKA promotes tumor progression by enhancing cell cycle progression, cell survival, genomic instability, epithelial-mesenchymal transition (EMT) and stem-like properties of cancer cells. ${ }^{[37]}$ In most solid tumors, AURKA regulates cell cycle checkpoints and promotes the cell cycle process. ${ }^{[37]}$ The GSEA based on GSE47911 gene profiles further validated the association between AURKA overexpression and cell cycle progression in GISTs.

To confirm the importance of AURKA expression in GISTs, we performed IHC staining to establish the associations between AURKA expression and clinicopathological characteristics of the enrolled 49 GIST patients. In advanced GISTs, the expression level of AURKA was found to be elevated. This result is consistent with the analyses of the data provided by GSE136755 and Lagarde et al. ${ }^{[24]}$ Survival analysis further showed that AURKA overexpression was a potential independent prognostic factor for GIST patients. Furthermore, a series of in vitro experiments demonstrated that GIST cells with overexpressed AURKA had significant malignance advantages, such as cell proliferation, anti-apoptosis and cell migration. The findings validated the results of bioinformatics analyses.

Drug resistance is a major obstacle in cancer chemotherapy and greatly affects a patient's prognosis. Adjuvant imatinib has been widely used as a first-line therapeutic option for advanced GIST patients ${ }^{[38,}$ ${ }^{39]}$. However, imatinib resistance has increased in recent years. By analyzing the raw data provided by Lagarde et al. ${ }^{[24]}$, a significant relationship was found between AURKA overexpression and gene mutations causing imatinib resistance. The GSE136755 data showed a similar but not significant result, which could be attributed to the small sample size. In addition, in vitro experiments showed that AURKA overexpression enhanced resistance of GIST cells to imatinib by promoting cell proliferation and inhibiting cell apoptosis.

The role of AURKA overexpression in tumor progression has been reported in a variety of human malignancies. AURKA phosphorylates RPS6KB1 and promotes cell proliferation as well as survival ${ }^{[40]}$. AURKA also stabilizes the transcription factor N-MYC, thereby promoting G1/S cell cycle transition and tumor cell proliferation ${ }^{[41]}$. Pharmacological inhibition of AURKA promotes the chemosensitivity of cervical cancer cells ${ }^{[42]}$. Compounds targeting AURKA, particularly alisertib, have been extensively studied in preclinical models, where they have shown synergistic effects with other targeted therapies, leading to tumor regression in a variety of cancer models ${ }^{[43]}$. Yeh et al. confirmed the contribution of the AURKA 
inhibitor, MLN8237, on the suppression of metastatic GISTs ${ }^{[44]}$. Findings from GEPIA and Oncomine databases also supported contribution of AURKA overexpression to tumorigenesis.

This study still had the following limitations. First, the case number in each GIST cohort is not large. Therefore, we made up for this deficiency to some extent by incorporating different GIST cohorts for comprehensive analysis. Second, this study is the preliminary exploration and certification of AURKA as a therapeutic target. As for the potential of AURKA-targeting therapy, we have not carried out experiments for investigation. Further validation based on various in vitro and in vivo experiments needs to be performed.

\section{Conclusions}

In conclusion, our findings demonstrated the significant overexpression of AURKA in advanced GISTs by bioinformatics analyses, which predicts a poor prognosis of GIST patients. Overexpression of AURKA was experimentally demonstrated to promote cell proliferation and inhibit cell apoptosis of GIST cells, which contributes to imatinib resistance, implying the potential of AURKA as a therapeutic target for GISTs.

\section{Declarations}

\section{Ethical approval and consent to participate}

This study was approved by the Ethical Committee of the First Affiliated Hospital, Zhejiang University School of Medicine.

\section{Consent for publication}

Not applicable.

\section{Availability of data and materials}

All data and materials generated and analyzed during this study are included in the main paper.

\section{Competing interests}

The authors declare no conflict of interest.

\section{Funding}

This research was supported by Zhejiang Provincial Natural Science Foundation of China under Grant No. LBY21H160005.

\section{Authors' contributions}


XC and WW, study conception and design, writing, review, and revision of the manuscript; XC and JW, bioinformatics analyses; WF and SL, in vitro experiments; XC, data acquisition, analysis and interpretation. All authors read and approved the final manuscript.

\section{Acknowledgement}

We acknowledge and appreciate Prof. Wenbin Chen for donating the GIST/T1 cell line.

\section{References}

1. Corless CL, Barnett CM, Heinrich MC. Gastrointestinal stromal tumours: origin and molecular oncology. Nat Rev Cancer. 2011;11:865-78.

2. Wozniak A, Gebreyohannes YK, Debiec-Rychter M, Schoffski P. New targets and therapies for gastrointestinal stromal tumors. Expert Rev Anticancer Ther. 2017;17:1117-29.

3. Liu P, Tan F, Liu H, Li B, Lei T, Zhao X. The Use of Molecular Subtypes for Precision Therapy of Recurrent and Metastatic Gastrointestinal Stromal Tumor. Onco Targets Ther. 2020;13:2433-47.

4. Joensuu $\mathrm{H}$. Risk stratification of patients diagnosed with gastrointestinal stromal tumor. Hum Pathol. 2008;39:1411-9.

5. Iorio N, Sawaya RA, Friedenberg FK. Review article: the biology, diagnosis and management of gastrointestinal stromal tumours. Aliment Pharmacol Ther. 2014;39:1376-86.

6. Valsangkar N, Sehdev A, Misra S, Zimmers TA, O'Neil BH, Koniaris LG. Current management of gastrointestinal stromal tumors: Surgery, current biomarkers, mutations, and therapy. Surgery. 2015;158:1149-64.

7. Serrano C, George S, Valverde C, Olivares D, Garcia-Valverde A, Suarez C, et al. Novel Insights into the Treatment of Imatinib-Resistant Gastrointestinal Stromal Tumors. Target Oncol. 2017;12:277-88.

8. Zhao X, Xu M, Cai Z, Yuan W, Cui W, Li MD. Identification of LIFR, PIK3R1, and MMP12 as Novel Prognostic Signatures in Gallbladder Cancer Using Network-Based Module Analysis. Frontiers in Oncology 2019;9.

9. Yang JF, Shi SN, Xu WH, Qiu YH, Zheng JZ, Yu K, et al. Screening, identification and validation of CCND1 and PECAM1/CD31 for predicting prognosis in renal cell carcinoma patients. Aging. 2019;11:12057-79.

10. Zheng H, Zhang G, Zhang L, Wang Q, Li H, Han Y, et al. Comprehensive Review of Web Servers and Bioinformatics Tools for Cancer Prognosis Analysis. Front Oncol. 2020;10:68.

11. Ohshima K, Fujiya K, Nagashima T, Ohnami S, Hatakeyama K, Urakami K, et al. Driver gene alterations and activated signaling pathways toward malignant progression of gastrointestinal stromal tumors. Cancer Sci. 2019;110:3821-33.

12. Barrett T, Wilhite SE, Ledoux P, Evangelista C, Kim IF, Tomashevsky M, et al. NCBI GEO: archive for functional genomics data sets-update. Nucleic Acids Res. 2013;41:D991-5. 
13. Huang da W, Sherman BT, Lempicki RA. Systematic and integrative analysis of large gene lists using DAVID bioinformatics resources. Nat Protoc. 2009;4:44-57.

14. Ashburner M, Ball CA, Blake JA, Botstein D, Butler H, Cherry JM, et al. Gene ontology: tool for the unification of biology. The Gene Ontology Consortium. Nat Genet. 2000;25:25-9.

15. Gene Ontology C. The Gene Ontology resource: enriching a GOld mine. Nucleic Acids Res. 2021;49:D325-34.

16. Kanehisa M. Toward understanding the origin and evolution of cellular organisms. Protein Sci. 2019;28:1947-51.

17. Kanehisa M, Furumichi M, Sato Y, Ishiguro-Watanabe M, Tanabe M. KEGG: integrating viruses and cellular organisms. Nucleic Acids Res. 2021;49:D545-51.

18. Szklarczyk D, Morris JH, Cook H, Kuhn M, Wyder S, Simonovic M, et al. The STRING database in 2017: quality-controlled protein-protein association networks, made broadly accessible. Nucleic Acids Res. 2017;45:D362-8.

19. Szklarczyk D, Gable AL, Lyon D, Junge A, Wyder S, Huerta-Cepas J, et al. STRING v11: protein-protein association networks with increased coverage, supporting functional discovery in genome-wide experimental datasets. Nucleic Acids Res. 2019;47:D607-13.

20. Shannon P, Markiel A, Ozier O, Baliga NS, Wang JT, Ramage D, et al. Cytoscape: a software environment for integrated models of biomolecular interaction networks. Genome Res. 2003;13:2498-504.

21. Ramos PIP, Arge LWP, Lima NCB, Fukutani KF, de Queiroz ATL. Leveraging User-Friendly Network Approaches to Extract Knowledge From High-Throughput Omics Datasets. Front Genet. 2019;10:1120.

22. Rhodes DR, Kalyana-Sundaram S, Mahavisno V, Varambally R, Yu J, Briggs BB, et al. Oncomine 3.0: genes, pathways, and networks in a collection of 18,000 cancer gene expression profiles. Neoplasia. 2007;9:166-80.

23. Tang Z, Li C, Kang B, Gao G, Li C, Zhang Z. GEPIA: a web server for cancer and normal gene expression profiling and interactive analyses. Nucleic Acids Res. 2017;45:W98-102.

24. Lagarde P, Perot G, Kauffmann A, Brulard C, Dapremont V, Hostein I, et al. Mitotic checkpoints and chromosome instability are strong predictors of clinical outcome in gastrointestinal stromal tumors. Clin Cancer Res. 2012;18:826-38.

25. Subramanian A, Tamayo P, Mootha VK, Mukherjee S, Ebert BL, Gillette MA, et al. Gene set enrichment analysis: a knowledge-based approach for interpreting genome-wide expression profiles. Proc Natl Acad Sci U S A. 2005;102:15545-50.

26. Mootha VK, Lindgren CM, Eriksson KF, Subramanian A, Sihag S, Lehar J, et al. PGC-1alpharesponsive genes involved in oxidative phosphorylation are coordinately downregulated in human diabetes. Nat Genet. 2003;34:267-73.

27. Lee EJ, Kang G, Kang SW, Jang KT, Lee J, Park JO, et al. GSTT1 copy number gain and ZNF overexpression are predictors of poor response to imatinib in gastrointestinal stromal tumors. PLoS 
One. 2013;8:e77219.

28. Chen WB, Cheng XB, Ding W, Wang YJ, Chen D, Wang JH, et al. Centromere protein F and survivin are associated with high risk and a poor prognosis in colorectal gastrointestinal stromal tumours. J Clin Pathol. 2011;64:751-5.

29. Schneider CA, Rasband WS, Eliceiri KW. NIH Image to ImageJ: 25 years of image analysis. Nat Methods. 2012;9:671-5.

30. Corless CL, Schroeder A, Griffith D, Town A, McGreevey L, Harrell P, et al. PDGFRA mutations in gastrointestinal stromal tumors: frequency, spectrum and in vitro sensitivity to imatinib. J Clin Oncol. 2005;23:5357-64.

31. von Mehren M, Joensuu H. Gastrointestinal Stromal Tumors. J Clin Oncol. 2018;36:136-43.

32. Agaimy A, Wunsch PH. Lymph node metastasis in gastrointestinal stromal tumours (GIST) occurs preferentially in young patients < or $=40$ years: an overview based on our case material and the literature. Langenbecks Arch Surg. 2009;394:375-81.

33. Joensuu H, Hohenberger P, Corless CL. Gastrointestinal stromal tumour. Lancet. 2013;382:973-83.

34. Keung EZ, Fairweather M, Raut CP. The Role of Surgery in Metastatic Gastrointestinal Stromal Tumors. Curr Treat Options Oncol. 2016;17:8.

35. Chen JS, Yeh CN, Cheng CT, Yen CC, Chen YY, Huang SC, et al. Role of PLK1 signaling pathway genes in gastrointestinal stromal tumors. Oncol Lett. 2018;16:3070-82.

36. Amirnasr A, Gits CMM, van Kuijk PF, Smid M, Vriends ALM, Rutkowski P, et al. Molecular Comparison of Imatinib-Naive and Resistant Gastrointestinal Stromal Tumors: Differentially Expressed microRNAs and mRNAs. Cancers (Basel) 2019;11.

37. Yan M, Wang C, He B, Yang M, Tong M, Long Z, et al. Aurora-A Kinase: A Potent Oncogene and Target for Cancer Therapy. Med Res Rev. 2016;36:1036-79.

38. Dematteo RP, Ballman KV, Antonescu CR, Maki RG, Pisters PW, Demetri GD, et al. Adjuvant imatinib mesylate after resection of localised, primary gastrointestinal stromal tumour: a randomised, doubleblind, placebo-controlled trial. Lancet. 2009;373:1097-104.

39. Joensuu H, Eriksson M, Sundby Hall K, Reichardt A, Hartmann JT, Pink D, et al. Adjuvant Imatinib for High-Risk GI Stromal Tumor: Analysis of a Randomized Trial. J Clin Oncol. 2016;34:244-50.

40. Wang-Bishop L, Chen Z, Gomaa A, Lockhart AC, Salaria S, Wang J, et al. Inhibition of AURKA Reduces Proliferation and Survival of Gastrointestinal Cancer Cells With Activated KRAS by Preventing Activation of RPS6KB1. Gastroenterology. 2019;156:662-75.e7.

41. Otto T, Horn S, Brockmann M, Eilers U, Schuttrumpf L, Popov N, et al. Stabilization of N-Myc is a critical function of Aurora A in human neuroblastoma. Cancer Cell. 2009;15:67-78.

42. Sun JM, Yang LN, Xu H, Chang B, Wang HY, Yang G. Inhibition of Aurora A promotes chemosensitivity via inducing cell cycle arrest and apoptosis in cervical cancer cells. Am J Cancer Res. 2015;5:1133-45. 
43. Otto T, Sicinski P. Cell cycle proteins as promising targets in cancer therapy. Nat Rev Cancer. 2017;17:93-115.

44. Yeh CN, Yen CC, Chen YY, Cheng CT, Huang SC, Chang TW, et al. Identification of aurora kinase A as an unfavorable prognostic factor and potential treatment target for metastatic gastrointestinal stromal tumors. Oncotarget. 2014;5:4071-86.

\section{Tables}

Table 1 Sequences of primers used for Quantitative real-time PCR analysis.

\begin{tabular}{|c|c|c|}
\hline Gene & Forward (5囚-3邓) & Reverse (5囚-3ð) \\
\hline Homo AURKA & TGGGTGGTCAGTACATGCTC & TGCATCCGACCTTCAATCATTTC \\
\hline Homo ACTB & AGCGAGCATCCCCCAAAGTT & GGGCACGAAGGCTCATCATT \\
\hline
\end{tabular}

Table 2 Correlation between AURKA expression level and clinical features in 49 GIST patients

\begin{tabular}{|c|c|c|c|c|c|}
\hline Parameter & Characteristic & No. patients (\%) & AURKA score & statistics & $P$ value \\
\hline \multirow[t]{2}{*}{ Gender } & Female & $23(46.9)$ & $1.93 \pm 1.35$ & $t=-0.430$ & 0.669 \\
\hline & Male & $26(53.1)$ & $2.12 \pm 1.52$ & & \\
\hline \multirow[t]{2}{*}{ Age } & $<57$ & $24(49.0)$ & $2.05 \pm 1.52$ & $t=-0.121$ & 0.904 \\
\hline & $\geq 57$ & $25(51.0)$ & $2.00 \pm 1.36$ & & \\
\hline \multirow[t]{3}{*}{ Location } & Stomach & $14(28.6)$ & $1.74 \pm 1.30$ & $F=1.321$ & 0.277 \\
\hline & Small intestine & $12(24.5)$ & $1.68 \pm 1.30$ & & \\
\hline & Large intestine & $23(46.9)$ & $2.37 \pm 1.53$ & & \\
\hline \multirow[t]{4}{*}{ Risk* } & High & $18(36.7)$ & $2.02 \pm 1.43$ & $F=35.449$ & $<0.001$ \\
\hline & Intermediate & $4(8.2)$ & $2.1 \pm 1.29$ & & \\
\hline & Low & $20(40.8)$ & $1.26 \pm 0.74$ & & \\
\hline & Very low & $7(14.3)$ & $0.40 \pm 0.52$ & & \\
\hline
\end{tabular}

${ }^{*} \mathrm{P}<0.05$ was considered statistically significant

Table 3 Correlation between AURKA intensity and clinical features based on GSE136755 


\begin{tabular}{|c|c|c|c|c|c|}
\hline Parameter & Characteristic & No. patients (\%) & AURKA intensity & statistics & $P$ value \\
\hline \multirow[t]{2}{*}{ Gender } & Female & $26(40.0)$ & $6.19 \pm 0.97$ & $t=-1.458$ & 0.150 \\
\hline & Male & $39(60.0)$ & $6.63 \pm 1.47$ & & \\
\hline \multirow[t]{2}{*}{ Age } & $<68$ & $31(47.7)$ & $6.28 \pm 1.17$ & $t=1.022$ & 0.311 \\
\hline & $\geq 68$ & $34(52.3)$ & $6.62 \pm 1.41$ & & \\
\hline \multirow[t]{3}{*}{ Location* } & Stomach & $43(66.2)$ & $6.14 \pm 1.25$ & $F=4.306$ & 0.018 \\
\hline & Non-stomach & $16(24.6)$ & $6.95 \pm 0.99$ & & \\
\hline & Metastasis & $6(9.2)$ & $7.39 \pm 1.74$ & & \\
\hline \multirow[t]{4}{*}{ Risk* } & High & $17(26.2)$ & $7.54 \pm 1.30$ & $F=11.898$ & $<0.001$ \\
\hline & Intermediate & $9(13.8)$ & $5.75 \pm 0.54$ & & \\
\hline & Low & $22(33.8)$ & $6.06 \pm 0.94$ & & \\
\hline & Very low & $11(16.9)$ & $5.66 \pm 0.74$ & & \\
\hline \multirow[t]{4}{*}{ Stage* } & IV & $9(13.8)$ & $7.94 \pm 1.63$ & $F=10.686$ & $<0.001$ \\
\hline & III & $9(13.8)$ & $6.85 \pm 0.89$ & & \\
\hline & $\|$ & $8(12.3)$ & $7.13 \pm 1.55$ & & \\
\hline & I & $39(60.0)$ & $5.89 \pm 0.84$ & & \\
\hline \multirow[t]{3}{*}{ Mutation } & sensitive & $56(86.2)$ & $6.39 \pm 1.21$ & $F=2.541$ & 0.087 \\
\hline & resistance & $7(10.8)$ & $7.33 \pm 1.84$ & & \\
\hline & $\mathrm{N} / \mathrm{A}$ & $2(3.1)$ & $5.29 \pm 0.20$ & & \\
\hline \multirow[t]{2}{*}{ Mutation } & Sensitive & $56(88.9)$ & $6.39 \pm 1.21$ & $t=1.814$ & 0.075 \\
\hline & Resistance & $7(11.1)$ & $7.33 \pm 1.84$ & & \\
\hline
\end{tabular}

$* \mathrm{P}<0.05$ was considered statistically significant 
Table 4 Correlation between AURKA intensity and clinical features based on the published raw data

\begin{tabular}{|c|c|c|c|c|c|}
\hline Parameter & Characteristic & No. patients (\%) & AURKA intensity & statistics & $P$ value \\
\hline \multirow[t]{3}{*}{ Location } & Stomach & $40(66.7)$ & $8.91 \pm 1.25$ & $F=1.923$ & 0.156 \\
\hline & Non-stomach & $16(26.7)$ & $9.64 \pm 1.55$ & & \\
\hline & Parenteral & $4(6.7)$ & $9.47 \pm 1.69$ & & \\
\hline \multirow[t]{4}{*}{ AFIP Risk* } & High & $17(28.3)$ & $10.2 \pm 1.59$ & $F=8.686$ & $<0.001$ \\
\hline & Intermediate & $14(23.3)$ & $9.38 \pm 1.43$ & & \\
\hline & Low & $16(26.7)$ & $8.48 \pm 0.64$ & & \\
\hline & Very low & $13(21.7)$ & $8.34 \pm 0.55$ & & \\
\hline \multirow[t]{2}{*}{ Local recurrence* } & No & $54(90.0)$ & $8.83 \pm 1.03$ & $t=7.377$ & $<0.001$ \\
\hline & Yes & $6(10.0)$ & $12.0 \pm 0.63$ & & \\
\hline \multirow[t]{2}{*}{ Metastasis* } & No & $45(75.0)$ & $8.56 \pm 0.82$ & $t=6.921$ & $<0.001$ \\
\hline & Yes & $15(25.0)$ & $10.9 \pm 1.22$ & & \\
\hline \multirow[t]{2}{*}{ Mutation* } & Sensitive & $45(75.0)$ & $8.91 \pm 0.18$ & $t=2.442$ & 0.018 \\
\hline & Resistance & $15(25.0)$ & $9.88 \pm 0.44$ & & \\
\hline
\end{tabular}

${ }^{*} \mathrm{P}<0.05$ was considered statistically significant

\section{Figures}


A

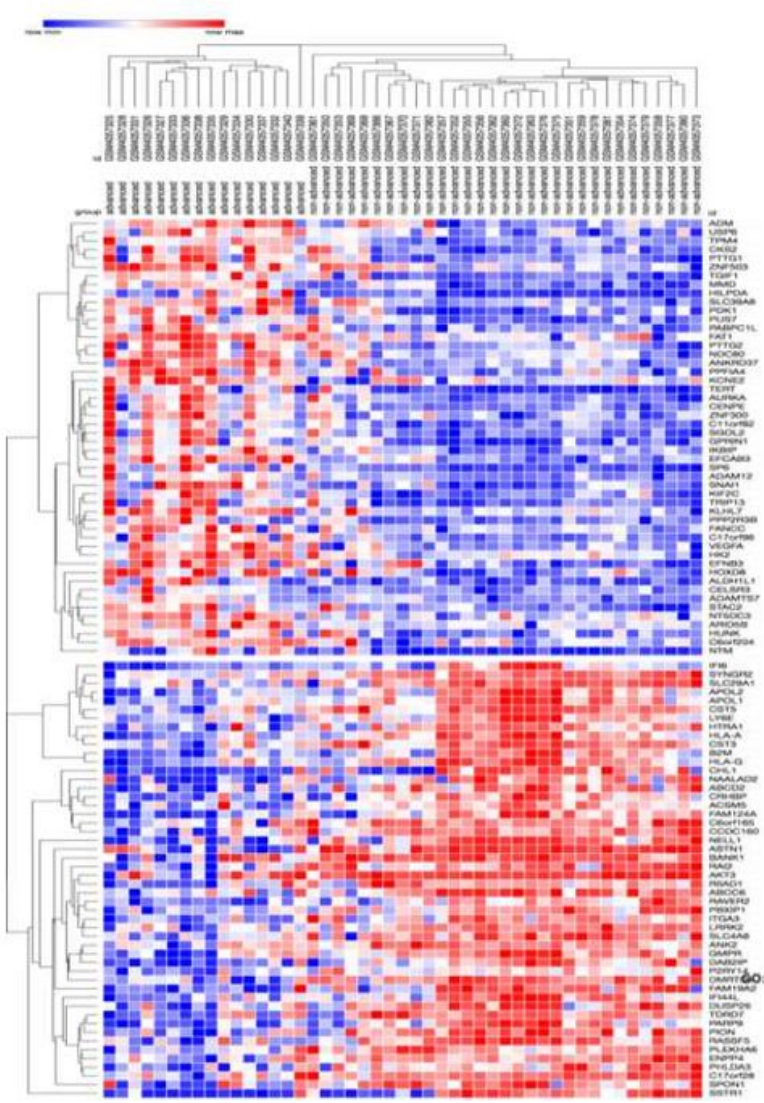

B
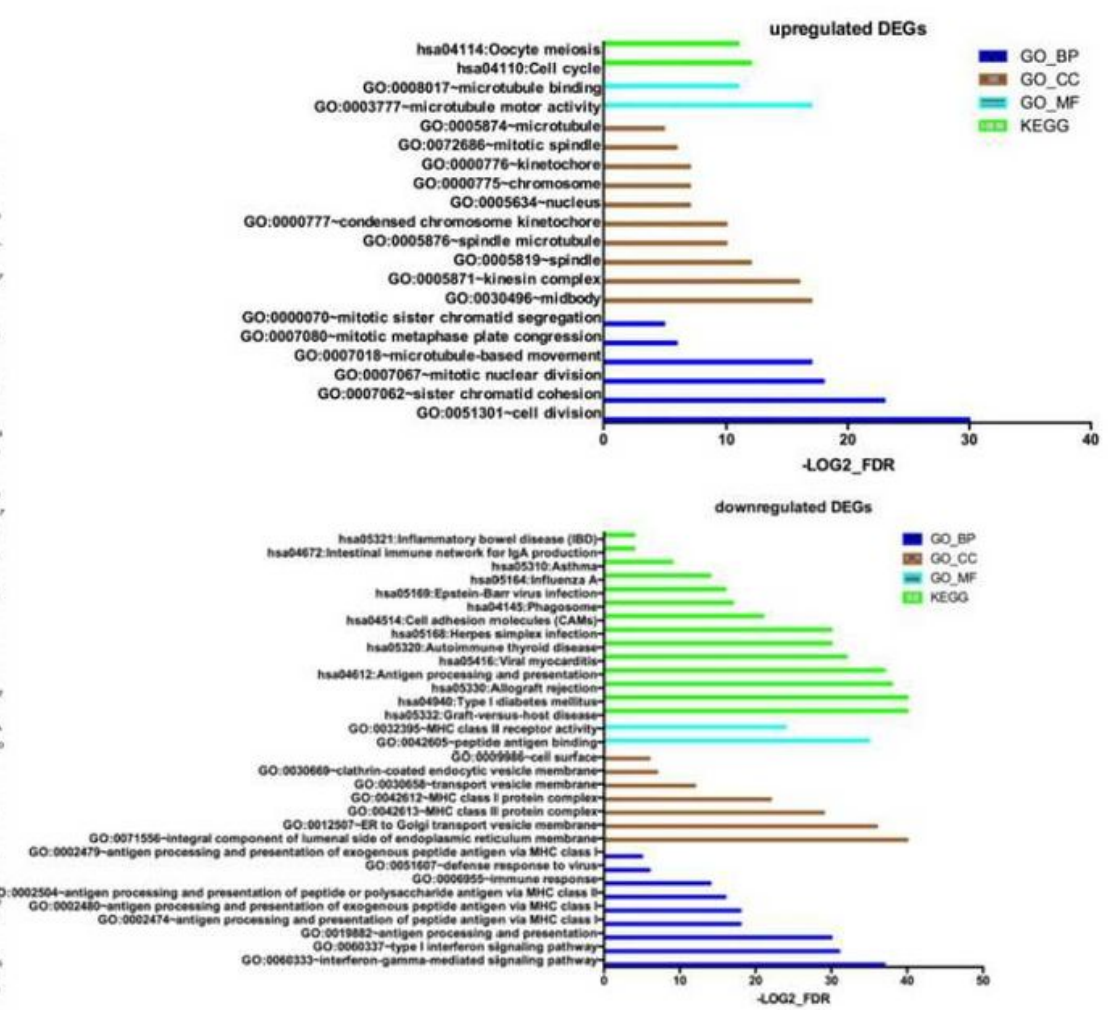

Figure 1

Screening of differentially expressed genes (DEGs) from the dataset GSE136755. (A) Heat map generated based on the top 50 up- and down-regulated DEGs between advanced and non-advanced GIST samples.

(B) Functional annotation of DEGs by GO and KEGG enrichment analyses. 

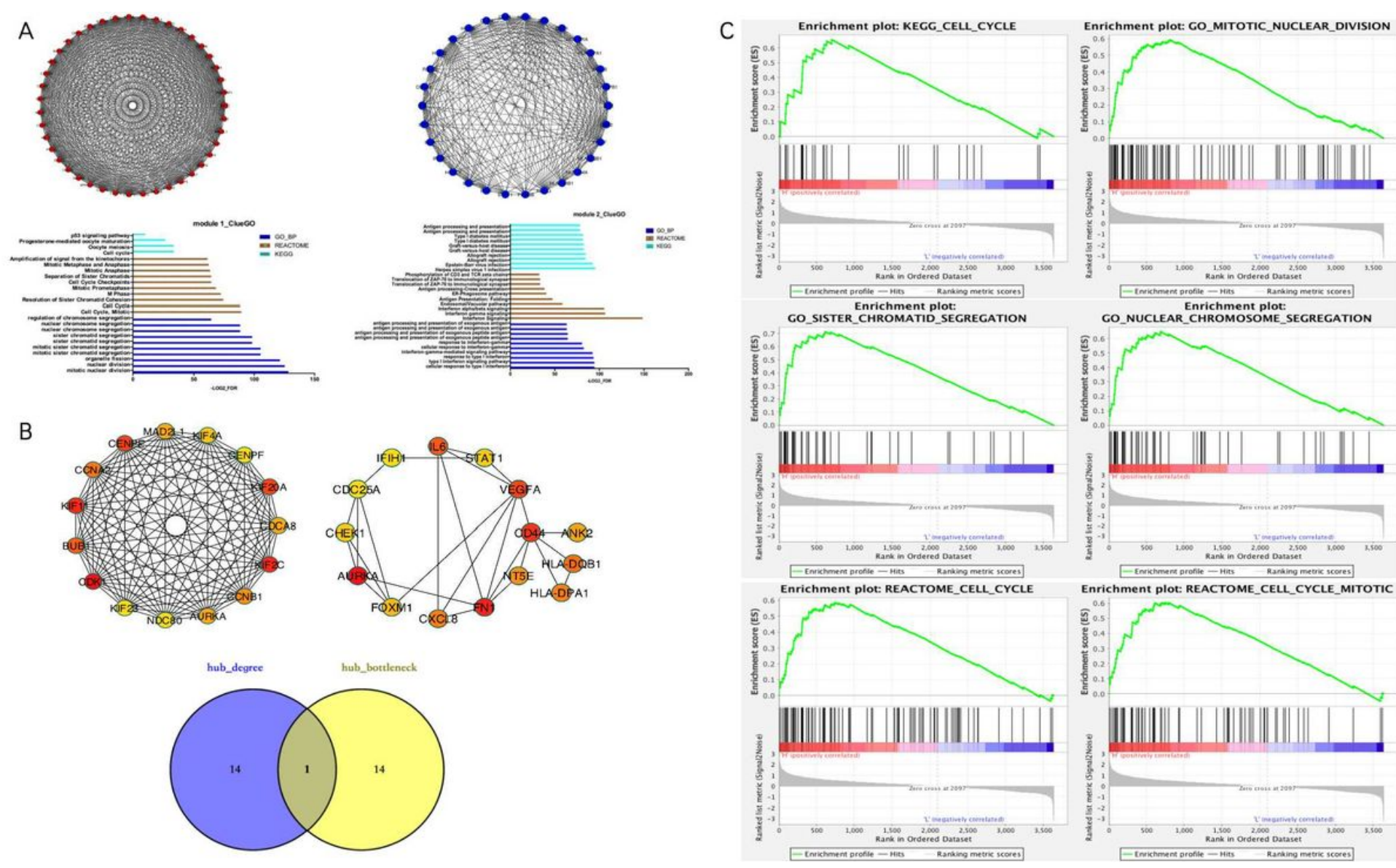

\section{Figure 2}

Identification of AURKA as a key hub gene in GISTs. (A) and (B) Based on dataset GSE136755, the most significant modules based on the topology in the protein-protein interaction (PPI) network were identified using Molecular Complex Detection (MCODE) of Cytoscape. Module 1 contains upregulated DEGs (red color), while module 2 contains downregulated DEGs (blue color). Functional annotation of modules 1 and 2 was determined by ClueGO. Hub genes were identified using CytoHubba of Cytoscape based on the degree and bottleneck algorithms. The key hub gene (AURKA) was obtained by taking the intersection of the two groups of hub genes. (C) Based on dataset GSE47911, gene set enrichment analysis (GSEA) results showed cell cycle related gene enrichment in AURKA elevated expression GISTs. 


\section{A}

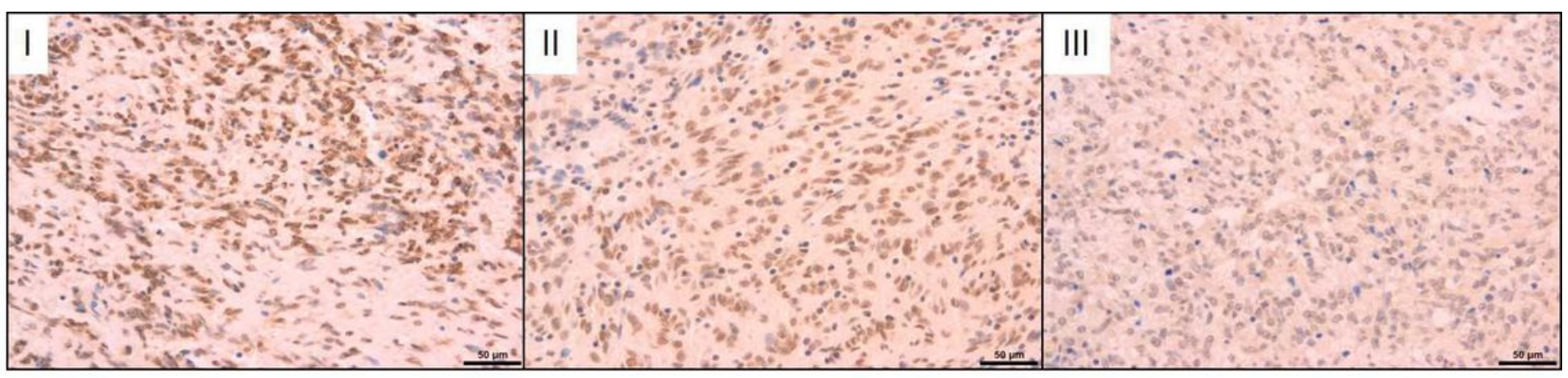

B

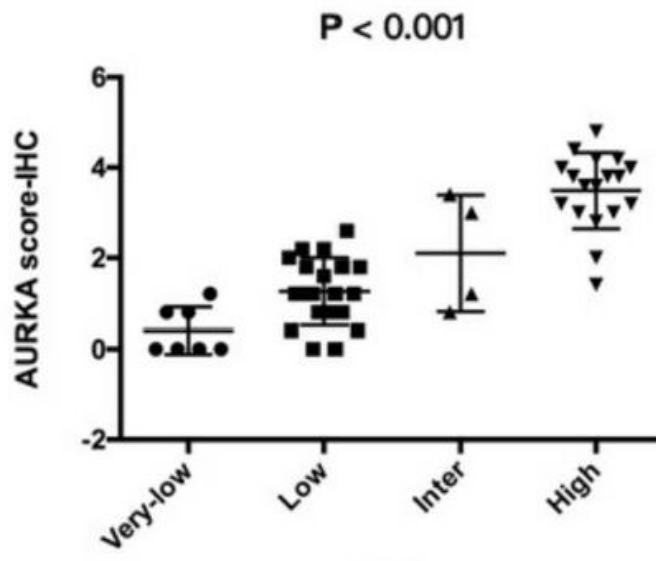

Risk

AURKA IHC staining from our hospital
C

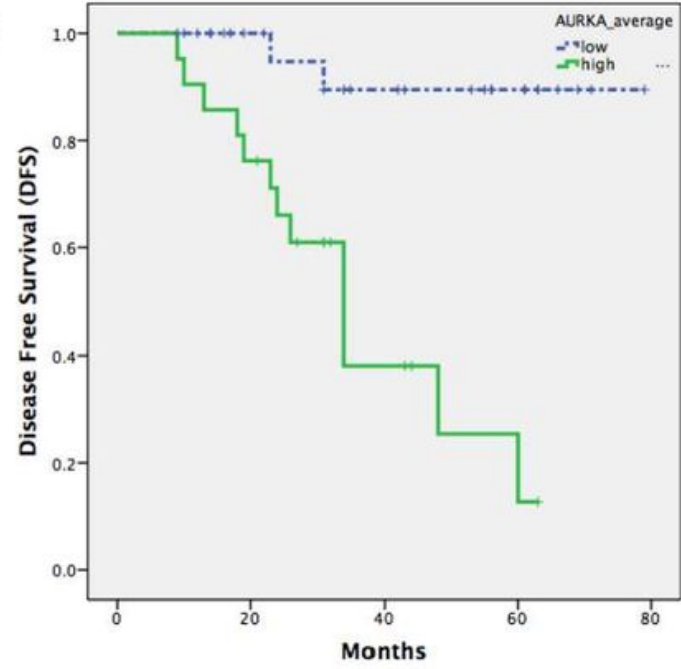

D
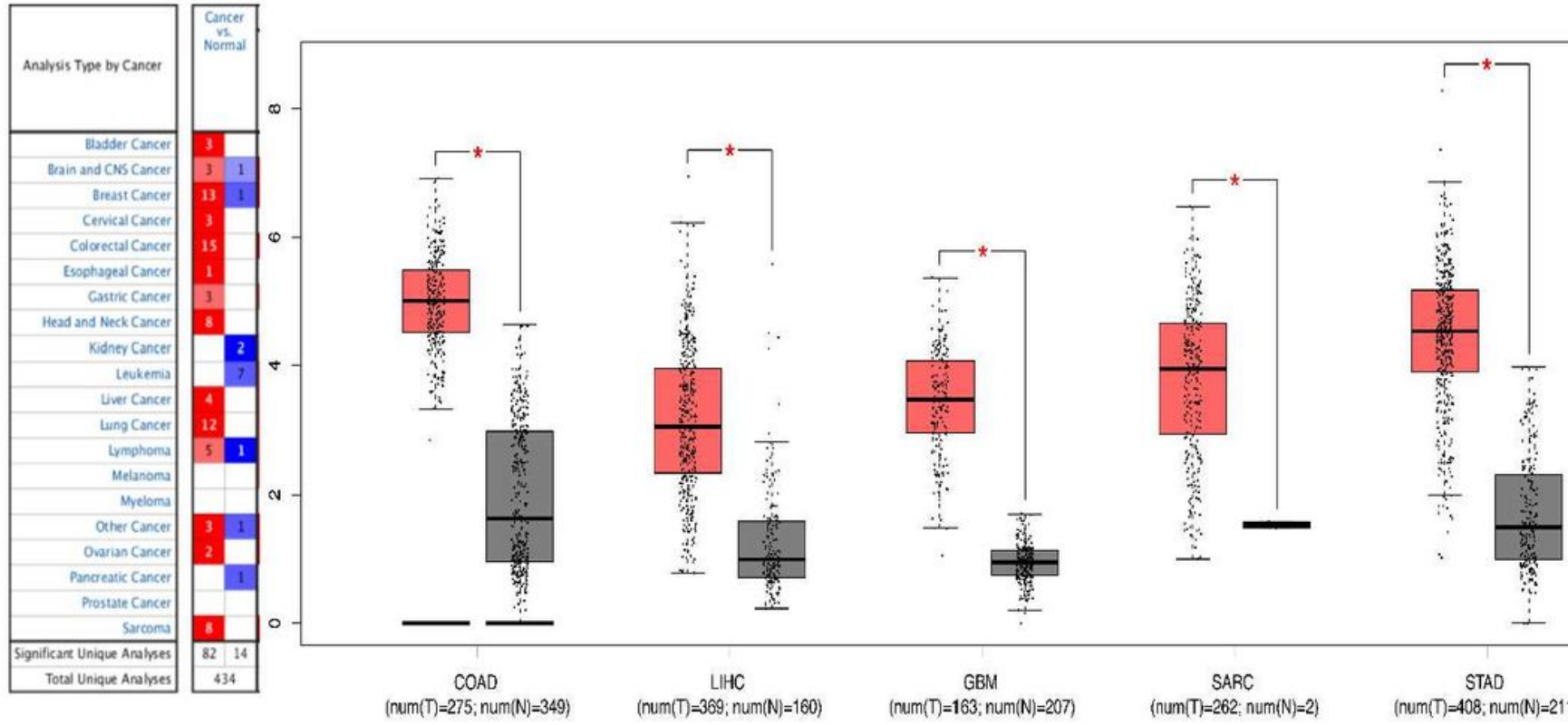

(num(T) $=369 ;$ num $(N)=160$ )

(num(T) $=163 ;$ num $(\mathrm{N})=207$ )

(num $(T)=262 ; \operatorname{num}(\mathrm{N})=2$ )

STAD

(num $(T)=408 ; \operatorname{num}(\mathrm{N})=211$ )

\section{Figure 3}

Expression patterns of AURKA in GISTs and other human malignancies. (A) Immunohistochemical (IHC) staining of GIST specimens. I: strong staining, II: intermediate staining, III: weak staining. (B) IHC scores of AURKA varied across different GIST risk groups, with high-risk GISTs showing the highest expression of AURKA. (C) Patients with elevated AURKA expression levels exhibited poorer DFS than those with low 
AURKA expression levels. (D) The Oncomine and GEPIA databases showed a significant upregulation of AURKA expression in most human malignancies by comparison to the normal tissues.

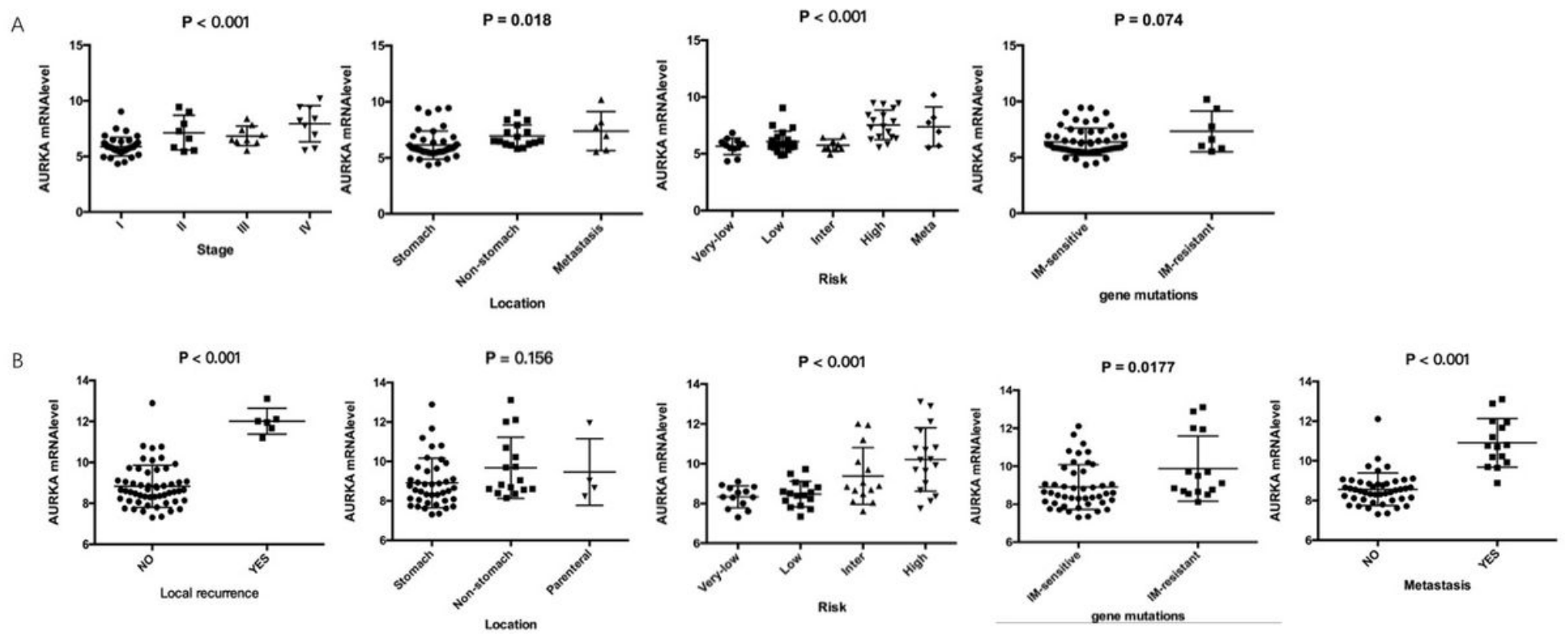

\section{Figure 4}

Expression profiles of AURKA in different GIST cohorts. (A) Based on data from the dataset GSE136755, AURKA expression was significantly associated with tumor location, stage and risk stratification, while there was no obvious correlation between AURKA expression and imatinib-related gene mutations. (B) Analysis of the raw data from Lagarde et al. (PMID: 22167411) revealed that AURKA expression was significantly associated with risk stratification and imatinib-related gene mutations. There is also an obvious correlation between AURKA expression and clinical outcomes, such as local recurrence and metastasis. There were no significant differences on AURKA expression levels among gastric, non-gastric and parenteral GISTs. 
A
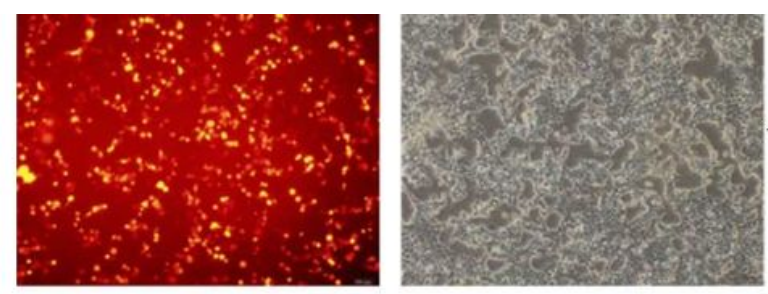

AURKA
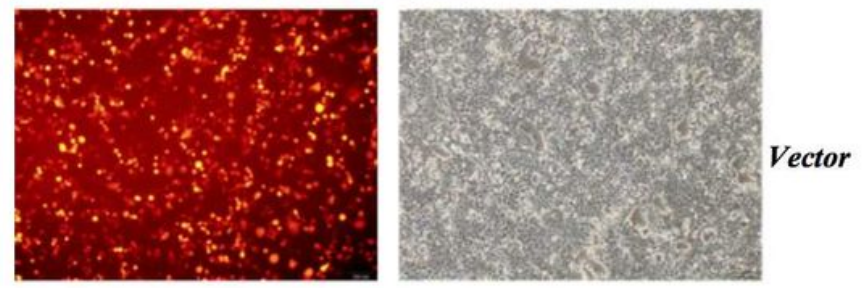

$$
P<0.001
$$

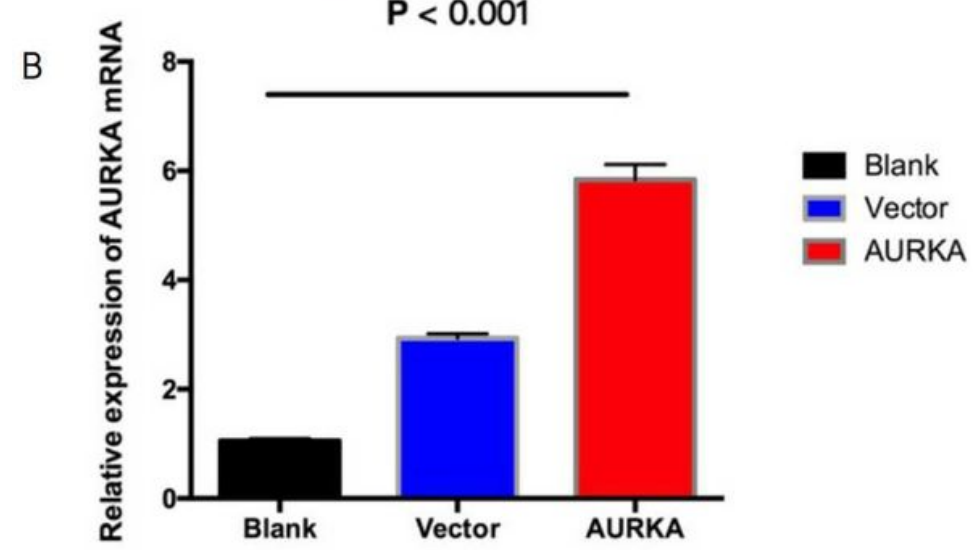

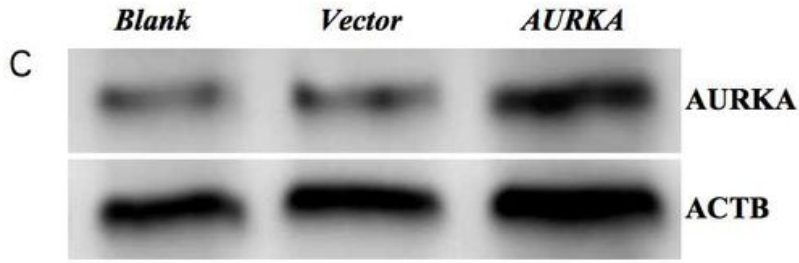

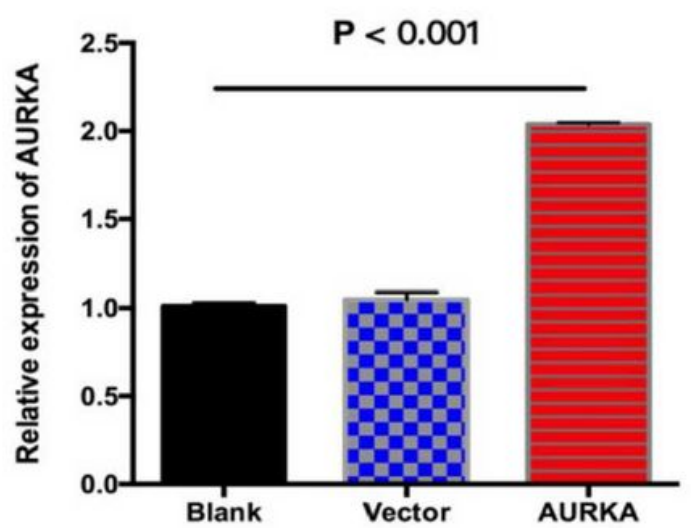

Figure 5

Construction of GIST/T1 cells with overexpressed AURKA. (A) Representative images of cells transfected with AURKA plasmids and control plasmids captured with fluorescent and bright field microscopy. (B) mRNA expression levels of AURKA are significantly higher in the AURKA group compared to the Blank and Vector groups. (C) Protein expression levels of AURKA are elevated in the AURKA group than in the Blank and Vector groups. 

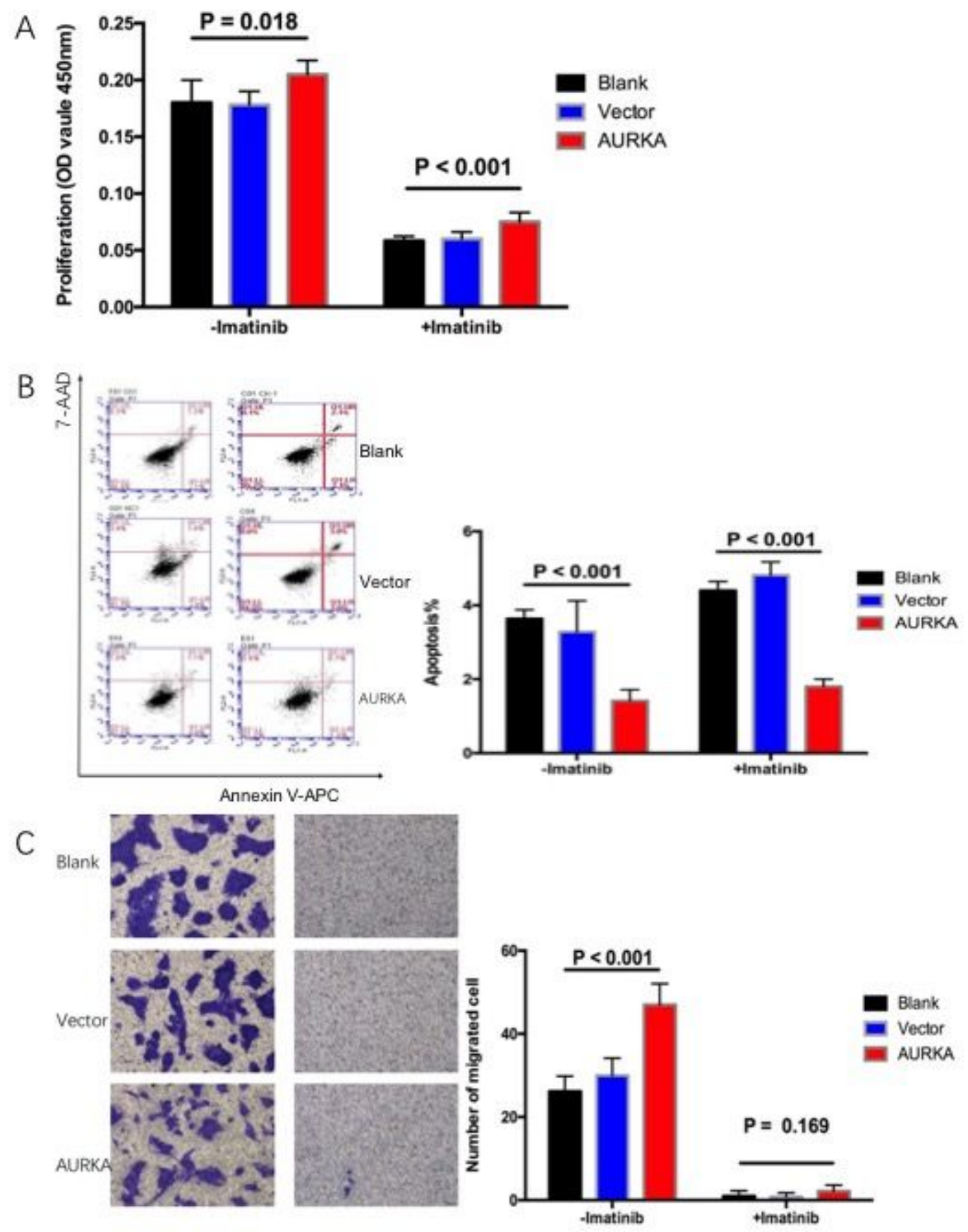

Figure 6

The effect of AURKA overexpression on the aggressive behaviors of GIST cells. (A) AURKA overexpression significantly promoted cell proliferation of GIST/T1 cells. (B) Annexin V assay revealed that AURKA overexpression significantly promoted cell survival and enhanced imatinib resistance in GIST/T1 cells. (C) Representative images of crystal violet staining showing the number of migrating cells in the Blank, Vector, and AURKA groups. AURKA overexpression significantly promoted GIST/T1 cell migration while this contribution could be eliminated by imatinib treatment. 